\title{
Functionalization of carbon-based nanostructures with light transition-metal atoms for hydrogen storage
}

\author{
E. Durgun, ${ }^{1,2}$ S. Ciraci, ${ }^{1,2, *}$ and T. Yildirim ${ }^{3,4}$ \\ ${ }^{1}$ Department of Physics, Bilkent University, Ankara 06800, Turkey \\ ${ }^{2}$ National Nanotechnology Research Center, Bilkent University, Ankara 06800, Turkey \\ ${ }^{3}$ NIST Center for Neutron Research, National Institute of Standards and Technology, Gaithersburg, Maryland 20899, USA \\ ${ }^{4}$ Department of Materials Science and Engineering, University of Pennsylvania, Philadelphia, Pennsylvania 19104, USA
}

(Received 5 October 2006; revised manuscript received 30 September 2007; published 4 February 2008)

In a recent letter [T. Yildirim and S. Ciraci, Phys. Rev. Lett. 94, 175501 (2005)], the unusual hydrogen storage capacity of Ti decorated carbon nanotubes has been revealed. The present paper extends this study further to investigate the hydrogen uptake by light transition-metal atoms decorating various carbon-based nanostructures in different types of geometry and dimensionality, such as carbon linear chain, graphene, and nanotubes. Using first-principles plane-wave method we show that not only outer but also inner surface of a large carbon nanotube can be utilized to bind more transition-metal atoms and hence to increase the storage capacity. We also found that scandium and vanadium atoms adsorbed on a carbon nanotube can bind up to five hydrogen molecules. Similarly, light transition-metal atoms can be adsorbed on both sides of graphene and each adsorbate can hold up to four hydrogen molecules yielding again a high-storage capacity. Interestingly, our results suggest that graphene can be considered as a potential high-capacity $\mathrm{H}_{2}$ storage medium. We also performed transition state analysis on the possible dimerization of $\mathrm{Ti}$ atoms adsorbed on the graphene and single-wall carbon nanotube.

DOI: 10.1103/PhysRevB.77.085405

PACS number(s): 73.63.Nm, 72.25.-b, 75.75.+a

\section{INTRODUCTION}

The development of fuel-cell technologies based on hydrogen holds the promise of producing clean and renewable energy. An efficient and safe storage of hydrogen is crucial for the advancement of hydrogen energy economy. ${ }^{1}$ Currently, a lot of effort has been devoted to engineer nanomaterials which can absorb hydrogen molecules with high gravimetric ${ }^{2}\left(g_{d}\right)$ and volumetric density, but release the stored hydrogens easily in the course of consumption., ${ }^{1,3-11}$ Much work has focused on carbon-based materials such as nanotubes, ${ }^{4-14} \mathrm{C}_{60},{ }^{15,16}$ metal hydrides, and metal-organic frameworks, ${ }^{17,18}$ titanium metallocarbohedryne, ${ }^{19,20}$ and polyacethylene. ${ }^{21}$ The main obstacles in hydrogen storage are slow kinetics, poor reversibility, and high dehydrogenation temperatures for chemical hydrides. ${ }^{17}$ Owing to their high surface-volume ratio, single- and multiwall carbon nanotubes appear to be suitable nanostructures for $\mathrm{H}_{2}$ storage. It is now well established that the interaction between hydrogen molecule and bare carbon nanotube or graphite surface is too weak, so that the absorbed molecules cannot remain attached at ambient temperature. ${ }^{13}$ In contrast, transition-metal (TM) atoms, such as $\mathrm{Pt}$ and $\mathrm{Pd}$, can bind multiple $\mathrm{H}_{2}$ molecules. ${ }^{13}$ Under certain circumstances, the adsorbed $\mathrm{H}_{2}$ can even dissociate and make strong TM-H bonds. From state-of-the-art first-principles calculations, it has been revealed that a single Ti atom adsorbed on a $(8,0)$ zigzag single-walled carbon nanotube (SWNT) can strongly bind up to four $\mathrm{H}_{2}$ molecules. ${ }^{14}$ At high Ti coverage it is found that a $(8,0)$ SWNT can store hydrogen molecules up to $g_{d}=0.078$ (or $\sim 8 \mathrm{wt} \%$ ), exceeding the minimum requirement of 6 wt \% set for practical applications. Similarly, it has been found that single $\mathrm{Ti}$ atom adsorbed on $\mathrm{C}_{60}$ can bind up to four hydrogen molecules yielding up to $\sim 8 \mathrm{wt} \%$ hydro- gen storage in case of full Ti coverage. ${ }^{15,16}$ However, it is argued that the storage capacity and hence the reversibility of the system are decreased because of the tendency of dimerization of $\mathrm{Ti}$ atom at high coverage. ${ }^{22}$ Very recently, it has been shown that a small molecule such as ethylene $\left(\mathrm{C}_{2} \mathrm{H}_{4}\right)$ can be complexed by light TM atoms to form an important basis for efficient and high-capacity medium for $\mathrm{H}_{2}$ coverage. ${ }^{23}$

In this paper, we extended the earlier work published as a short letter ${ }^{14}$ to other carbon-based structures functionalized by TM atoms such as $\mathrm{Sc}$, Ti, and V. We considered carbonbased structures in three different types of geometry; onedimensional (1D) linear carbon atomic chain $\mathrm{C}_{n}$ ( $n$ being the number of carbon atoms in the chain), two-dimensional (2D) graphene, and tubular SWNT with relatively larger radius. To increase the storage capacity further, we examined the possibility of $\mathrm{H}_{2}$ uptake of a single TM atom adsorbed on the inner wall of the $(12,0)$ SWNT. We found that two light TM atoms can be adsorbed concomitantly to external (convex) and internal (concave) walls of a SWNT, and they can hold up to eight $\mathrm{H}_{2}$ molecules at the same time. This result indicates a significant raise of the gravimetric density of $\mathrm{H}_{2}$ stored by SWNTs. Remarkably, two Ti atoms each capping one free end of a linear segment of carbon atomic chain $\mathrm{C}_{n}$ to form Ti- $\mathrm{C}_{n}$-Ti complex can hold up to ten $\mathrm{H}_{2}$ and attain a storage capacity of $14.4 \mathrm{wt} \%$ for $n=2$. Two light TM atoms adsorbed on both sides, namely, one adsorbed above and the other below the center of a same hexagonal site, can bind up to eight $\mathrm{H}_{2}$ molecules. This way a stable $(2 \times 2)$ pattern can be formed with the storage capacity of $7.8 \mathrm{wt} \%$. The tendency for dimerization of TM atoms at high coverage has been examined for SWNT as well as graphene. Our results are of particular importance, since graphene has been synthesized recently and its properties have been a growing subject of interest. 
The paper is organized as follows: In Sec. II we describe the computational method that we used in this study. In Sec. III we study linear carbon chain-TM interactions and their hydrogen absorption properties. We discuss hydrogen absorption properties of TM decorated graphene layer in Sec. IV. The adsorption of TM atoms on the external and internal walls of the $(12,0)$ SWNT and their hydrogen uptake is discussed in Sec. V. The energetics associated with the dimerization of Ti atoms, which are adsorbed on graphene or SWNT surface, is investigated in Sec. VI. Our conclusions are summarized in Sec. VII.

\section{METHOD}

We have performed first-principles plane-wave calculations ${ }^{24,25}$ within density functional theory ${ }^{26}$ using ultrasoft pseudopotentials. ${ }^{25,27}$ The exchange correlation potential has been approximated by generalized gradient approximation (GGA) using PW91 functional ${ }^{28}$ both for spinpolarized and spin-unpolarized cases. Following electronic states are treated as valence: Ti: $3 s^{2} 3 p^{6} 3 d^{2}\left(3 s^{2} 3 p^{6} 3 d^{1}\right.$ for Sc and $3 s^{2} 3 p^{6} 3 d^{3}$ for $\left.\mathrm{V}\right) 4 s^{2} ; \mathrm{C}: 2 s^{2} 2 p^{2}$; and $\mathrm{H}: 1 s$. For partial occupancies we use the Methfessel-Paxton smearing method. ${ }^{29}$ All structures have been treated within a supercell geometry using the periodic boundary conditions. A spacing $\sim 10 \AA$ between nearest TM atoms in adjacent cells has been left to prevent structures from their coupling. In the selfconsistent potential and total energy calculations the Brillouin zone of graphene and SWNT is sampled in the $\mathbf{k}$ space within Monkhorst-Pack scheme ${ }^{30}$ by $(12 \times 12 \times 1)$ and $(1 \times 1 \times 15)[(1 \times 1 \times 11)$ for double cell $]$ mesh points, respectively. A plane-wave basis set with kinetic energy of $350 \mathrm{eV}$ has been used. All atomic positions and lattice parameters are optimized by using the conjugate gradient method where total energy and atomic forces are minimized. The convergence for energy is chosen as $10^{-5} \mathrm{eV}$ between two ionic steps, and the maximum force allowed on each atom is $0.05 \mathrm{eV} / \AA$.

\section{LINEAR CARBON CHAIN}

The simplest carbon-based structure, namely, a 1D, finitesize carbon chain, $\mathrm{C}_{n}$, has been synthesized ${ }^{31,32}$ recently and studied extensively. ${ }^{33}$ Earlier, it has been shown that a segment of carbon chain comprising $n$ atoms has a stable and linear structure owing to the double bonds between two adjacent $\mathrm{C}$ atoms ${ }^{33}$ Its ideal and periodic form is metallic with conductance $G=4 e^{2} / h$. Here, we first investigate $\mathrm{Ti}^{-\mathrm{C}_{5}}$ finite structure (one end of the carbon chain is capped with $\mathrm{Ti}$, while the other end is left free and ready to be attached to any other structure having high surface to volume ratio like a SWNT). We found that the interaction between $\mathrm{Ti}$ and $\mathrm{C}_{5}$ is quite strong and has the binding energy ${ }^{34} E_{b}=4.1 \mathrm{eV}$. Here, Ti atom can absorb up to five $\mathrm{H}_{2}$ molecules with an average binding energy of $0.42 \mathrm{eV}$ [see Fig. 1(a)]. The ground state of Ti- $\mathrm{C}_{5}$ is magnetic with $\mu=4 \mu_{B}$, but it becomes nonmagnetic when the Ti atom is saturated by five $\mathrm{H}_{2}$ molecules. Another absorption state with a comparable total energy is the configuration where the first $\mathrm{H}_{2}$ is dissociated but the

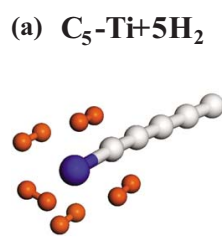

$\mathrm{E}_{\mathrm{b}}=0.42 \mathrm{eV}$

$\mathrm{g}_{\mathrm{d}}=0.085$ (b) $\mathrm{Ti}-\mathrm{C}_{3}-\mathrm{Ti}+10 \mathrm{H}_{2}$

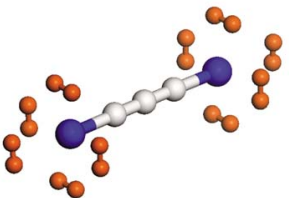

$\mathrm{E}_{\mathrm{b}}=0.55 \mathrm{eV}$

$\mathrm{g}_{\mathrm{d}}=0.133$ (c) $\mathrm{Ti}-\mathrm{C}_{2}-\mathrm{Ti}+10 \mathrm{H}_{2}$

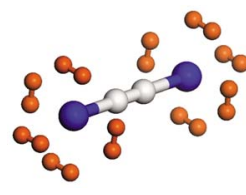

$\mathrm{E}_{\mathrm{b}}=0.47 \mathrm{eV}$

$\mathrm{g}_{\mathrm{d}}=0.144$
FIG. 1. (Color online) (a) Final optimized atomic structure of a linear Ti- $\mathrm{C}_{5}$ complex holding five $\mathrm{H}_{2}$ with average binding energy $E_{b}=0.42 \mathrm{eV}$ per $\mathrm{H}_{2}$ and gravimetric density $g_{d}=0.085$. (b) Same for $\mathrm{C}_{3}$ chain capped by two Ti atoms from both ends holding ten $\mathrm{H}_{2}$ with $g_{d}=0.133$. (c) Same for $\mathrm{C}_{2}$ molecule with $g_{d}=0.144$ (or $14.4 \mathrm{wt} \%)$. Respectively, small, medium, and large balls indicate $\mathrm{H}, \mathrm{C}$, and $\mathrm{Ti}$ atoms.

remaining four $\mathrm{H}_{2}$ are molecularly adsorbed with $\mathrm{H}-\mathrm{H}$ bonds slightly elongated to $\sim 0.8 \AA$.

As an alternative structure, we now consider another Ti atom that saturates the free end of $\mathrm{Ti}^{-} \mathrm{C}_{n}$ to form Ti- $\mathrm{C}_{n}$-Ti. Then, this complex can absorb altogether ten $\mathrm{H}_{2}$ molecules and has $g_{d}=20 M_{\mathrm{H}} /\left(n M_{\mathrm{C}}+2 M_{\mathrm{Ti}}+20 M_{\mathrm{H}}\right)$ in terms of the atomic mass of hydrogen $\left(M_{\mathrm{H}}\right)$, carbon $\left(M_{\mathrm{C}}\right)$, and titanium $\left(M_{\mathrm{Ti}}\right)$. As shown in Figs. 1(b) and 1(c), the gravimetric density of stored $\mathrm{H}_{2}$ molecules becomes $g_{d}=0.133$ and 0.144 for $n=3$ and $n=2$, respectively. Apart from $\mathrm{C}$ atoms which are bound to $\mathrm{Ti}$ atoms at both ends, $\mathrm{C}$ atoms in the chain can also interact strongly with free $\mathrm{H}_{2}$ molecules at close proximity and dissociate them to form $\mathrm{C}-\mathrm{H}$ bonds. However, further functionalization through $\mathrm{Ti}$ atoms bridging other $\mathrm{C}=\mathrm{C}$ bonds makes the chain unstable. This is due to fact that small separation between $\mathrm{Ti}$ atoms leads to stronger $\mathrm{Ti}-\mathrm{Ti}$ coupling. At the end, linear chains are transformed to the clusters composed of $\mathrm{C}$ and $\mathrm{Ti}$ atoms.

Similar results are also obtained for Sc and V. For example, single $\mathrm{V}$ attached to a free end of the linear $\mathrm{C}_{n}$ can absorb also up to five $\mathrm{H}_{2}$ molecules with an average binding of $0.57 \mathrm{eV}$. Since V is heavier than Ti, the calculated storage capacity is slightly reduced to $8.3 \mathrm{wt} \%$. In closing, we note that the synthesis of $\mathrm{C}_{n}$ with controlled $n$ may not be feasible with today's technology; the present study is nevertheless valuable for the exploration of high-storage capacity medium based on small TM-C clusters.

\section{GRAPHENE}

Graphene, a single atomic layer of graphite, has a 2D hexagonal lattice. Hexagons form a "honeycomb" structure, since the primitive unit cell has two carbon atoms as basis. Earlier, graphene has been known as the atomic plane of graphite and also as the 2D lattice of $\mathrm{C}$ atoms which forms SWNT when it is wrapped on a cylinder. Recently, single graphene has been synthesized. The behavior of electrons as Dirac fermion with exceptional transport properties and also other properties has made graphene as the current subject of interest. ${ }^{35}$ Since individual graphene has been synthesized, its capacity of $\mathrm{H}_{2}$ absorption should be as interesting as carbon nanotubes or fullerenes. 
(a)

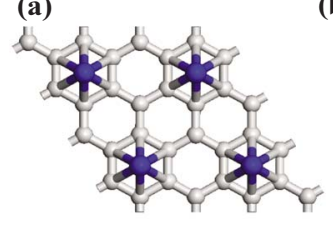

(b)

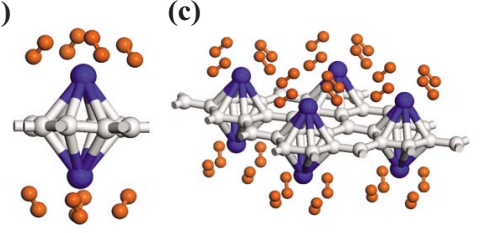

FIG. 2. (Color online) (a) A $(2 \times 2)$ pattern of Ti atoms occupying the hexagonal sites. (b) Two TM ( $\mathrm{Sc}, \mathrm{Ti}$, or $\mathrm{V}$ ) atoms shown by large dark ball adsorbed below and above the center of a hexagon of carbon atoms in graphene. Each TM atom binds up to four $\mathrm{H}_{2}$ molecules. (c) A $(2 \times 2)$ cell of graphene binding two $\mathrm{TM}+4 \mathrm{H}_{2}$ ligand; one above and the other below the atomic plane.

We consider a $(2 \times 2)$ unit cell (supercell) and one $\mathrm{Ti}$ atom is adsorbed above the center of a hexagon of carbon atoms in this unit cell. This pattern corresponds to the projection of the $\mathrm{Ti}$ decoration on the curved surface of $(8,0)$ SWNT used as low $\mathrm{Ti}$ coverage in Ref. 14. Accordingly, there is only one Ti per eight $\mathrm{C}$ atoms specified as $\mathrm{C}_{8}(\mathrm{TM})$ in the $(2 \times 2)$ unit cell as shown in Fig. 2(a). The nearest Ti-Ti interaction on this planar geometry is smaller than that on the curved geometry. The spin-polarized calculations show that Ti-graphene interaction is quite strong with a binding energy of $1.27 \mathrm{eV}$ per $\mathrm{Ti}$ atom. ${ }^{36}$ Note that $E_{b}$ calculated for graphene is smaller than that is calculated for carbon nanotube (see the next section and Refs. 37 and 38 due to curvature effect). ${ }^{8}$ Each Ti atom in Fig. 2(a) can bind up to four $\mathrm{H}_{2}$ molecules and hence $g_{d}=8 M_{\mathrm{H}} /\left(8 M_{\mathrm{H}}+8 M_{\mathrm{C}}+M_{\mathrm{Ti}}\right)$ becomes 0.053 . Interestingly, another $\mathrm{Ti}$ atom can be attached below the center of the same hexagon just below the first $\mathrm{Ti}$ as illustrated in Fig. 2(b). The binding energy of the second $\mathrm{Ti}$ atom is relatively smaller $\left(E_{b}=1.17 \mathrm{eV}\right)$. We found that this decoration of graphene by $\mathrm{Ti}$ atoms above and below the atomic plane to form a $(2 \times 2)$ pattern is stable. As $\mathrm{H}_{2}$ molecules are attaching one by one to these $\mathrm{Ti}$ atoms above and below, dissociation of $\mathrm{H}_{2}$ did not occur. Each Ti atom absorbs up to four $\mathrm{H}_{2}$ with an average binding energy of $0.35 \mathrm{eV}$ per $\mathrm{H}_{2}$. Hydrogen molecules are distributed almost symmetrically around $\mathrm{Ti}$ atoms with an average distance of $1.97 \AA$. The fifth $\mathrm{H}_{2}$ escaped without forming a physisorption bond with $\mathrm{Ti}$. In this particular geometry the interaction between $\mathrm{Ti}$ and six $\mathrm{C}$ atoms at the close proximity limits the storage capacity to $g_{d}=8 M_{\mathrm{H}} /\left(8 M_{\mathrm{H}}+4 M_{\mathrm{C}}+M_{\mathrm{Ti}}\right)$ yielding 7.8 wt $\%$.

Similar results are obtained by replacing Ti with either Sc or $\mathrm{V}$. The binding energy of Sc $(\mathrm{V})$ adsorbed at the hexagonal sites is calculated to be $1.49(0.95) \mathrm{eV}$. If two Sc (V) atoms are adsorbed above and below the center of the same hexagon keeping a $(2 \times 2)$ pattern, the binding energy of the second atom is $1.69(0.73) \mathrm{eV}$. It appears that $E_{b}$ decreases with increasing atomic number of adsorbed TM atom. Each Sc (V) atom can bind up to four $\mathrm{H}_{2}$ molecules with an average binding energy of $0.17(0.33) \mathrm{eV}$ per $\mathrm{H}_{2}$ and approximate storage capacity of $8.0(7.5) \mathrm{wt} \%$. The origin of this unusual binding of four $\mathrm{H}_{2}$ molecules for each TM atoms is explained by well-known Dewar ${ }^{39}$ coordination and Kubas ${ }^{40}$ interaction. The TM atoms are chemically bonded onto graphene through hybridization of lowest unoccupied mo- lecular orbital with TM $d$ orbitals (i.e., Dewar coordination). The resulting complex then binds multiple hydrogen molecules through hybridization between $\mathrm{H}_{2}-\sigma^{*}$ antibonding and TM $d$ orbital (i.e., Kubas interaction). Similar binding occurs for light TM atoms adsorbed on SWNT.

\section{SINGLE-WALL CARBON NANOTUBE}

In Ref. 14 we have considered Ti covered $(8,0)$ SWNT as a medium of high-capacity hydrogen storage. Here we will address the question how the radius, $r$, of a SWNT affects the storage capacity. To this end, in the previous section we treated the graphene corresponding to a SWNT with infinite radius. In this section we will consider the $(12,0)$ zigzag SWNT, which has a radius larger than that of $(8,0)$ tube. Larger radius also allows us to examine whether a $\mathrm{Ti}$ atom can be adsorbed on the internal wall of the SWNT, and whether this Ti atom by itself can bind $\mathrm{H}_{2}$ molecules.

We first answer to the question whether the radius of a nanotube affects the binding of Ti atoms. We found that the $\mathrm{Ti}$ atom favors the hexagonal site (above the center of the hexagon) and its binding energy was calculated to be $1.54 \mathrm{eV}$. Although the favorable adsorption site is the same for both $(8,0)$ and $(12,0)$ tubes, the binding energy of the latter (having larger radius, $r=4.7 \AA$ ) is approximately $0.6 \mathrm{eV}$ smaller than that of the $(8,0)$ tube $^{37,38}$ having $r$ $=3.2 \AA$. This curvature effect is expected, since C $2 p$ and $\mathrm{Ti} 3 d$ hybridizations are enhanced at small radius. The variation of the binding energy with radius is significant at small radius, but converges to zero as radius increases. The binding energy of Ti decreases from 2.2 to $1.27 \mathrm{eV}$ as the radius increases from $r=3.2 \AA$ to infinity.

We found that $\mathrm{Ti}$ atom that is adsorbed externally on the $(12,0)$ SWNT surface can bind up to four $\mathrm{H}_{2}$ in two different configurations (or absorption state). Both configurations have comparable total energies. In the first configuration, the first $\mathrm{H}_{2}$ is dissociated to form two strong Ti-H bonds with a $\mathrm{H}-\mathrm{H}$ distance much larger than that of $\mathrm{H}_{2}$. The remaining three $\mathrm{H}_{2}$ are molecularly absorbed with slightly elongated $\mathrm{H}-\mathrm{H}$ distance. According to the second configuration, four $\mathrm{H}_{2}$ molecules are absorbed around Ti symmetrically with an average binding energy of $0.56 \mathrm{eV}$ per $\mathrm{H}_{2}$ as described in Ref. 14. However, the total energy of the first configuration is $20 \mathrm{meV}$ lower (more energetic) than that of the second one. This situation was reversed in the case of $(8,0)$ SWNT, ${ }^{14}$ perhaps due to curvature effect. Nevertheless, the energy difference is small and is within the accuracy limits of the present calculations. The atomic configuration is illustrated in Fig. 3(a). To test the stability of the geometry we displaced $\mathrm{Ti}+4 \mathrm{H}_{2}$ ligand in the direction perpendicular to the wall of SWNT and also towards the C-C bond from the center of hexagon. After relaxation, displaced structures have returned to their initial equilibrium geometry.

It is noted that the average binding energy of four $\mathrm{H}_{2} \mathrm{kept}$ by a single $\mathrm{Ti}$ atom adsorbed on a $(12,0)$ zigzag tube is found to be close to that calculated for the $(8,0)$ tube. ${ }^{14}$

We now examine the adsorption of a single $\mathrm{Ti}$ atom on the internal wall of SWNT. The binding interaction between $\mathrm{Ti}$ and SWNT is quite strong and results in a binding energy of 
(a) $\mathrm{SWNT}+\mathrm{Ti}(\mathrm{ext})+2 \mathrm{H}+3 \mathrm{H}_{2}$

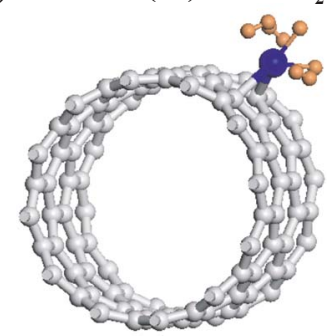

(b)

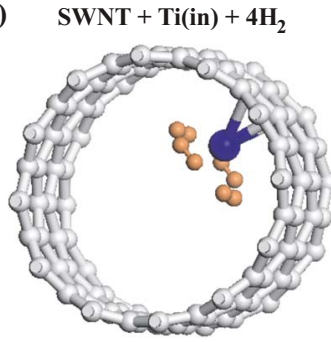

(c) $\mathrm{SWNT}+\mathrm{Ti}(\mathrm{in})+\mathrm{Ti}(\mathrm{ext})+8 \mathrm{H}_{2}$

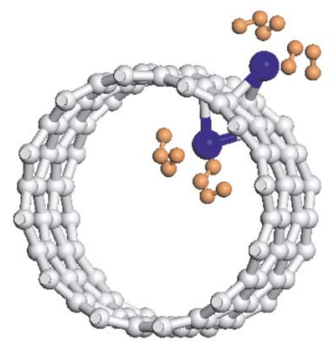

(d) $\mathrm{SWNT}+\mathrm{V}(\mathrm{ext})+\mathbf{5} \mathrm{H}_{2}$

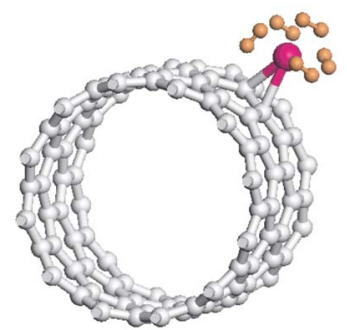

(e)

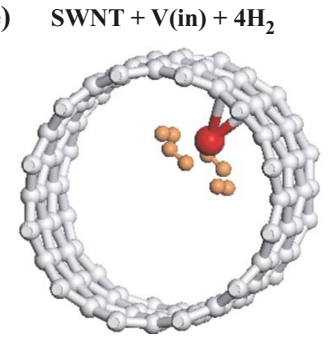

(f) $\mathrm{SWNT}+\mathrm{V}(\mathrm{in})+\mathrm{V}(\mathrm{ext})+8 \mathrm{H}_{2}$

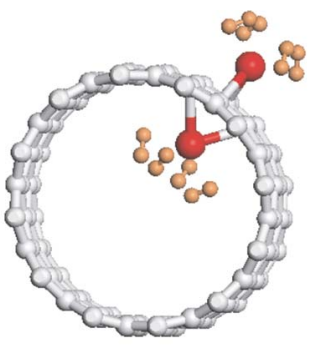

FIG. 3. (Color online) Atomic configuration of Ti atom adsorbed on the surface of the $(12,0)$ SWNT binding multiple $\mathrm{H}_{2}$ molecules: (a) $\mathrm{Ti}$ adsorbed externally (i.e., $\mathrm{Ti}_{e x}$ ) and holding four $\mathrm{H}_{2}$ one of which is dissociated. (b) $\mathrm{Ti}$ atom adsorbed internally (i.e., $\mathrm{Ti}_{\text {in }}$ ) and holding four $\mathrm{H}_{2}$. (c) Two Ti atoms adsorbed at the same time (i.e., $\mathrm{Ti}_{e x}$ and $\mathrm{Ti}_{i n}$ ). One $\mathrm{Ti}$ is adsorbed externally and holding four $\mathrm{H}_{2}$, and another Ti adsorbed internally and holding four $\mathrm{H}_{2}$. (d) Externally adsorbed $\mathrm{V}$ binding five $\mathrm{H}_{2}$. (e) Internally adsorbed $\mathrm{V}$ binding four $\mathrm{H}_{2}$. (f) $\mathrm{V}_{e x}$ and $\mathrm{V}_{\text {in }}$ each holding four $\mathrm{H}_{2}$ molecules.

$1.57 \mathrm{eV}$. Like the external case, internally absorbed $\mathrm{Ti}$ can bind also up to four $\mathrm{H}_{2}$ but with a relatively weak binding energy of $0.27 \mathrm{eV}$. The binding configuration is shown in Fig. 3(b). Dissociation of $\mathrm{H}_{2}$ did not occur even for the first molecule.

Next, we consider two Ti atoms adsorbed on the wall of the $(12,0)$ SWNT at the same time; while one Ti is adsorbed externally above the center of the hexagon of carbon atoms $\left(\mathrm{Ti}_{e x}\right)$, the other $\mathrm{Ti}$ is adsorbed internally below the first one $\left(\mathrm{Ti}_{\text {in }}\right)$. The average binding energy per $\mathrm{Ti}$ atom is close to the case of adsorption of single Ti atom. In this case, a total of seven $\mathrm{H}_{2}$ are absorbed $\left(\mathrm{Ti}_{e x}\right.$ binds up to four $\mathrm{H}_{2}$, and $\mathrm{Ti}_{\text {in }}$ binds only three $\mathrm{H}_{2}$ ), if the first $\mathrm{H}_{2}$ molecule attached to $\mathrm{Ti}_{e x}$ is dissociated. Otherwise, up to eight $\mathrm{H}_{2}$ can be absorbed (four $\mathrm{H}_{2}$ by $\mathrm{Ti}_{e x}$ and four $\mathrm{H}_{2}$ by $\mathrm{Ti}_{i n}$, if all $\mathrm{H}_{2}$ are placed around $\mathrm{Ti}$ atoms with well-defined symmetry as shown in Fig. 3.

Vanadium atom can be adsorbed externally above the center of hexagon on the wall of SWNT with binding energy of $E_{b}=0.93 \mathrm{eV}$. It can bind up to four $\mathrm{H}_{2}$ with an average bind- ing energy of $0.46 \mathrm{eV}$ per $\mathrm{H}_{2}$, if one of hydrogen molecules is dissociated. Otherwise, up to five $\mathrm{H}_{2}$ can be molecularly absorbed with an average binding energy of $0.40 \mathrm{eV}$ per $\mathrm{H}_{2}$, if all molecules are placed around $\mathrm{V}$ with a well-defined symmetry. ${ }^{41}$ The binding energy of the last (fifth) $\mathrm{H}_{2}$ is calculated as $0.16 \mathrm{eV}$. The atomic configurations of absorbed $\mathrm{H}_{2}$ molecules and $\mathrm{V}$ atoms are shown in Figs. 3(d)-3(f). We note that the distance between SWNT and adsorbed $\mathrm{V}$ atom $d_{\mathrm{V}-\mathrm{C}}$ increases as more $\mathrm{H}_{2}$ are bound to $\mathrm{V}$ due to more charge transferred from $\mathrm{C}-\mathrm{Ti}$ bond to $\mathrm{V}+\mathrm{H}_{2}$ bonds. This brings up the question of instability at high gravimetric density. To check the stability, we first pull the $\mathrm{V}+5 \mathrm{H}_{2}$ ligand from the surface of SWNT to $2.9 \AA$. Upon relaxation of the structure, the $\mathrm{V}+5 \mathrm{H}_{2}$ ligand has returned to its original, equilibrium structure. The energetics and relevant data for the atomic structure for $\mathrm{H}_{2}$ held by TM atoms adsorbed on carbon-based structures are summarized in Table I.

It should be noted that as shown in this section light TM atoms can be bound concomitantly to the external as well as to the internal wall of SWNT beyond a certain radius. For smaller radius, the increased coupling between nearest neighbor $\mathrm{Ti}$ atoms leads to dimerization of $\mathrm{Ti}$ atoms. This is an undesired situation. For example, Ti clusters form inside the $(12,0)$ tube for coverages of $\mathrm{TM}$ specified as $\mathrm{C}_{4}(\mathrm{TM})$ and $\mathrm{C}_{8}(\mathrm{TM})$. For relatively more dilute internal coverage of four $\mathrm{Ti}$ and two Ti per unit cell, the probability of clustering decreases. The storage capacity can be calculated for two different uniform coverage of TM externally adsorbed on SWNT; namely, $\mathrm{C}_{8}(\mathrm{TM})$ and $\mathrm{C}_{4}(\mathrm{TM})$. The low uniform coverage corresponds to $(2 \times 2)$ pattern on graphene where one hexagonal site is left unoccupied between two adsorbed TM atoms. For external adsorption of TM atoms, $g_{d}$ $=8 M_{\mathrm{H}} /\left(8 M_{\mathrm{H}}+8 M_{\mathrm{C}}+M_{\mathrm{TM}}\right)$ is calculated to be $0.054,0.053$, and 0.052 for $\mathrm{Sc}, \mathrm{Ti}$, and $\mathrm{V}$, respectively. If both external adsorption with $\mathrm{C}_{8}(\mathrm{TM})$ and internal adsorption with $\mathrm{C}_{16}(\mathrm{TM})$, one finds $g_{d}=80 M_{\mathrm{H}} /\left(80 M_{\mathrm{H}}+48 M_{\mathrm{C}}+10 M_{\mathrm{TM}}\right)$ to be $0.072,0.071$, and 0.069 for $\mathrm{Sc}$, Ti, and V.

For the high external coverage of TM atoms, namely, $\mathrm{C}_{4}(\mathrm{TM})$ (where $12 \mathrm{Ti}$ atoms are adsorbed on 12 hexagonal sites forming a ring perpendicular to the tube axis, but leaving a ring of empty hexagonal sites between the adjacent $\mathrm{Ti}$ rings), $g_{d}=8 M_{\mathrm{H}} /\left(8 M_{\mathrm{H}}+4 M_{\mathrm{C}}+M_{\mathrm{TM}}\right)$ is calculated to be $0.080,0.078$, and 0.075 for $\mathrm{Sc}, \mathrm{Ti}$, and $\mathrm{V}$, respectively. These are the common value for $(n, 0)$ zigzag and $(n, n)$ armchair nanotubes. In the next section, we will show that in $\mathrm{C}_{4}(\mathrm{Ti})$ coverage two adjacent $\mathrm{Ti}$ atoms favor the dimerization which reduces the storage capacity. If five $\mathrm{H}_{2}$ absorption by Sc and $\mathrm{V}$ is considered, $g_{d}$ is calculated to be 0.098 and 0.092 for $\mathrm{Sc}$ and $\mathrm{V}$, respectively.

Having discussed binding energies and corresponding gravimetric densities at high coverage, we now estimate the desorption temperature, $T_{D}$, using van't Hoff equation,

$$
T_{D}=\frac{E_{b}}{k_{B}}\left(\frac{\Delta S}{R}-\ln p\right)^{-1}
$$

in terms of $E_{b}$, Boltzmann constant $k_{B}$, change in $\mathrm{H}_{2}$ entropy from gas to liquid phase $\Delta S$, gas constant $R$, and equilibrium pressure of $1 \mathrm{~atm}$. Using $\Delta S$ from Ref. 42 and the average 
TABLE I. $E_{b}(\mathrm{TM})$ is calculated binding energy of a single $\mathrm{TM}$ atom $(\mathrm{TM}=\mathrm{Sc}, \mathrm{Ti}$, and $\mathrm{V})$ adsorbed to different carbon-based structures. $E_{b}\left(\mathrm{H}_{2}\right)$ is the average binding energy of $\mathrm{H}_{2}$ molecules absorbed by a single TM atom. $d_{\mathrm{TM}-\mathrm{H}}$ is average distance between TM and $\mathrm{H}_{2} \cdot d_{\mathrm{H}-\mathrm{H}}$ is the distance between two $\mathrm{H}$ atoms of molecularly absorbed $\mathrm{H}_{2}$. The hydrogen storage capacity in wt $\%$ of $\mathrm{TM}-\mathrm{C}_{5}, \mathrm{TM}_{2}$-graphene in $(2 \times 2)$ pattern, and $(12,0) \mathrm{SWNT}$. For the latter the first and second numerals stand for the low $\left[\mathrm{C}_{8}(\mathrm{TM})\right]$ and high $\left[\mathrm{C}_{4}(\mathrm{TM})\right] \mathrm{TM}$ coverages, respectively. Numbers in parentheses correspond to the coverage where five $\mathrm{H}_{2}$ are absorbed by a TM atom.

\begin{tabular}{|c|c|c|c|c|c|c|}
\hline & Structure & $E_{b}(\mathrm{TM})(\mathrm{eV})$ & $E_{b}\left(\mathrm{H}_{2}\right)$ & $d_{\mathrm{TM}-\mathrm{H}}(\AA)$ & $d_{\mathrm{H}-\mathrm{H}}$ & wt $\%$ \\
\hline \multirow[t]{5}{*}{$\mathrm{Sc}$} & $\mathrm{C}_{5}$ & 3.2 & 0.36 & 2.00 & 0.80 & 8.8 \\
\hline & Graphene & 1.59 & 0.17 & 2.01 & 0.82 & 8.0 \\
\hline & $(12,0)$ ext & 1.28 & $0.47(0.45)$ & 2.05 & 0.82 & $5.4-8.0(9.8)$ \\
\hline & $(12,0) \mathrm{int}$ & 1.37 & 0.39 & 2.06 & 0.82 & \\
\hline & $(12,0)$ ext-int & 1.53 & 0.31 & 2.06 & 0.83 & $7.2-9.1$ \\
\hline \multirow[t]{5}{*}{$\mathrm{Ti}$} & $\mathrm{C}_{5}$ & 4.12 & 0.42 & 1.9 & 0.81 & 8.5 \\
\hline & Graphene & 1.22 & 0.35 & 1.9 & 0.82 & 7.8 \\
\hline & $(12,0)$ ext & 1.54 & 0.56 & 1.9 & 0.82 & $5.3-7.8$ \\
\hline & $(12,0)$ int & 1.57 & 0.27 & 1.87 & 0.86 & \\
\hline & $(12,0)$ ext-int & 1.55 & 0.48 & 1.9 & 0.81 & $7.1-8.8$ \\
\hline \multirow[t]{5}{*}{ V } & $\mathrm{C}_{5}$ & 3.46 & 0.57 & 1.81 & 0.83 & 8.3 \\
\hline & Graphene & 0.84 & 0.32 & 1.77 & 0.86 & 7.5 \\
\hline & $(12,0)$ ext & 0.93 & $0.46(0.40)$ & 1.77 & 0.87 & $5.2-7.5(9.2)$ \\
\hline & $(12,0)$ int & 0.83 & 0.37 & 1.72 & 0.90 & \\
\hline & $(12,0)$ ext-int & 0.86 & 0.41 & 1.71 & 0.90 & $6.9-8.5$ \\
\hline
\end{tabular}

binding energy from Table I we can estimate the average desorption temperature $T_{D}$. By using the binding energy of the last $\mathrm{H}_{2}$ absorbed by TM, we can deduce the onset of the desorption at $T_{D_{L}}<T_{D}$. Similarly, by using the binding energy of the first $\mathrm{H}_{2}$ absorbed by TM we can also deduce highest temperature $T_{D_{H}}>T_{D}$ required for full discharge of hydrogen from the medium. In Table II we present the estimated $T_{D_{L}}, T_{D}$, and $T_{D_{H}}$ for the desorption of $\mathrm{H}_{2}$ absorbed by light TM atoms. ${ }^{43}$

\section{DIMERIZATION OF ADSORBED Ti ATOMS}

In a recent study Sun et al. ${ }^{22}$ pointed out that the hydrogen storage capacity of $\mathrm{Ti}$ covered $\mathrm{C}_{60}$ is reduced as a result of the dimerization of two $\mathrm{Ti}$ atoms adsorbed at hexagonal sites. Based on similar calculations they first found that the formation of $\mathrm{Ti}_{2}$ dimers is energetically favorable. Furthermore, they showed that two $\mathrm{H}_{2}$ out of eight $\mathrm{H}_{2}$ molecules absorbed to $\mathrm{Ti}_{2}$ dimer dissociate and hence storage capacity is reduced. Earlier, it was shown that the adjacent $\mathrm{Ti}$ atoms

TABLE II. The desorption temperatures of $\mathrm{H}_{2}$ molecules estimated by using van't Hoff equation absorbed by $\mathrm{Sc}, \mathrm{Ti}$, and $\mathrm{V}$ atoms attached to various carbon-based nanostructures (see text).

\begin{tabular}{|c|c|c|c|c|c|}
\hline & Structure & Average $E_{b}$ & $T_{D_{L}}(\min )$ & $T_{D}$ & $T_{D_{H}}(\max )$ \\
\hline \multirow[t]{4}{*}{$\mathrm{Sc}$} & Graphene & 0.17 & 124 & 217 & 391 \\
\hline & $(12,0)$ ext & 0.45 & 367 & 575 & 733 \\
\hline & $(12,0)$ int & 0.39 & 293 & 498 & 660 \\
\hline & $(12,0)$ ext-int & 0.31 & 196 & 396 & 562 \\
\hline \multirow[t]{4}{*}{$\mathrm{Ti}$} & Graphene & 0.35 & 244 & 447 & 611 \\
\hline & $(12,0)$ ext & 0.56 & 501 & 716 & 867 \\
\hline & $(12,0)$ int & 0.27 & 147 & 345 & 513 \\
\hline & $(12,0)$ ext-int & 0.48 & 403 & 613 & 770 \\
\hline \multirow[t]{4}{*}{ V } & Graphene & 0.32 & 210 & 410 & 570 \\
\hline & $(12,0)$ ext & 0.40 & 306 & 511 & 672 \\
\hline & $(12,0)$ int & 0.37 & 269 & 473 & 636 \\
\hline & $(12,0)$ ext-int & 0.41 & 318 & 524 & 684 \\
\hline
\end{tabular}




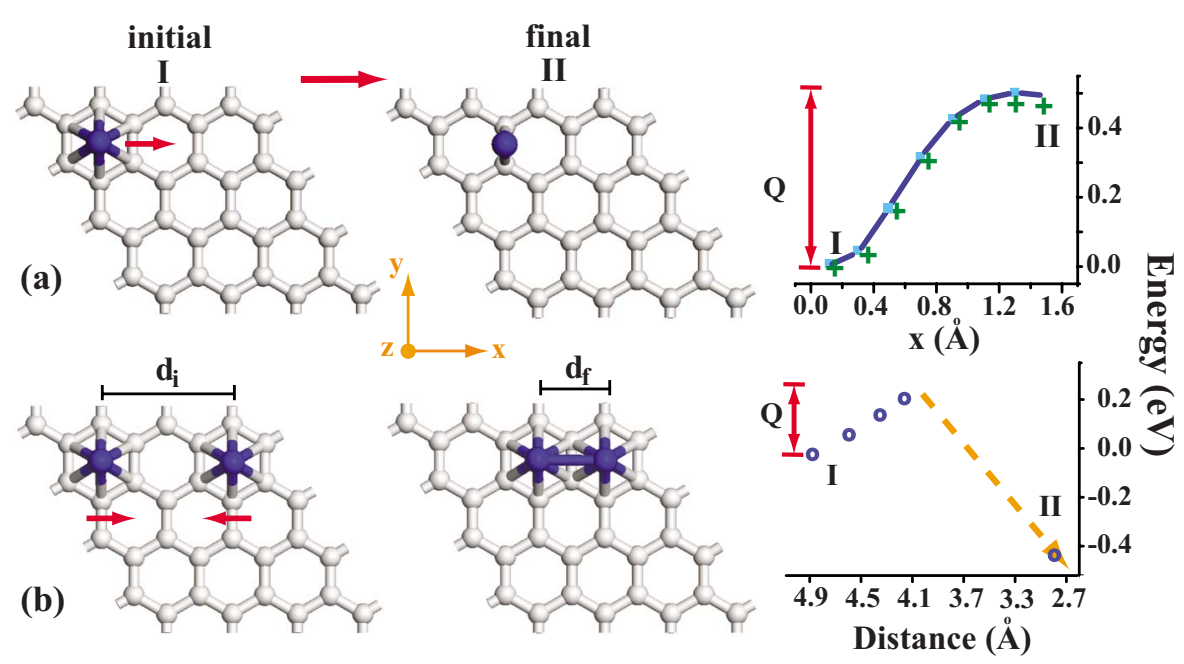

FIG. 4. (Color online) (a) Variation of total energy in transition of a single Ti atom from any hexagonal site of a $(4 \times 4)$ graphene to the bridge site and the involved energy barrier $Q$. Variations of total energy by fixing and relaxing the graphene atoms are indicated by square and crosses, respectively. (b) Variation of the total energy and involved energy barrier $Q$ in transition from two Ti atoms adsorbed at two hexagonal sites (leaving one empty hexagonal site between them) to a $\mathrm{Ti}_{2}$ dimer as a final state II. Here, while the left adsorbed $\mathrm{Ti}$ atoms is moved to the right in increments, its $y$ and $z$ coordinates and all the coordinates of the left Ti atom are relaxed. $d_{i}$ and $d_{f}$ are initial and final (after relaxation) distances between two Ti atoms. Carbon and $\mathrm{Ti}$ atoms are indicated by gray and dark balls, respectively.

favor the dimerization upon the relaxation of the initial, uniform $\mathrm{C}_{2}(\mathrm{Ti})$ coverage in which each hexagonal site is occupied by one Ti atom. ${ }^{44}$ Here we examine the tendency for the dimerization of $\mathrm{Ti}$ atoms adsorbed on a graphene and on the $(12,0)$ SWNT at different levels of coverage.

We first study the diffusion of a single Ti as a first step in any dimerization or clustering process. Since Ti atoms are preferably adsorbed at the hexagonal sites, we calculate the energy barrier as one Ti diffuses from one hexagonal site to the adjacent one. As a single $\mathrm{Ti}$ atom in $(4 \times 4)$ supercell is moved along the $x$ axis, its $y$ and $z$ coordinates and all atomic positions of underlying graphene are relaxed to minimize the total energy. In Fig. 4(a) we calculated the energy barrier $Q$ to be $\sim 0.5 \mathrm{eV}$ for the path from the hexagonal site to the minimum energy position at the bridge site. We note that the path from the hollow site to the top site has barrier energy $11 \mathrm{meV}$ higher than the bridge site. The phonon frequency of the adsorbed Ti atom is low due to its big mass and is estimated from the potential energy given in Fig. 4(a) as $\nu \simeq 3$ $\times 10^{12} \mathrm{~s}^{-1}$. The surface diffusion constant, $D=D_{0} e^{-Q / k_{B} T}$, $1.3 \times 10^{-3} \mathrm{~cm} \mathrm{~s}^{-1}$ is at room temperature, with $D_{0}=4.2$ $\times 10^{4} \mathrm{~cm} \mathrm{~s}^{-1}$. This value is considered to be small. While the binding energy of a single $\mathrm{Ti}$ atom is $1.27 \mathrm{eV}$, the binding of the second $\mathrm{Ti}$ to the adjacent hexagonal site is found to be $2.59 \mathrm{eV}$ in equilibrium. The coupling between two Ti atoms placed at the adjacent hexagonal site is very strong and leads to the formation of $\mathrm{Ti}_{2}$ dimer. We see that the gain of energy by dimer formation relative to the energy of two $\mathrm{Ti}$ atoms adsorbed already on two distant hexagonal sites is $\sim 1.32 \mathrm{eV}$. Sun et al. $^{22}$ found dimerization energy on $\mathrm{C}_{60}$ as $1.29 \mathrm{eV}$.

We now consider two $\mathrm{Ti}$ atoms adsorbed on the hexagonal sites of the graphene $(4 \times 4)$ supercell as shown in Fig. 4(b) as initial state I. While the first (left) $\mathrm{Ti}$ atom moves to the right in increments of $x$, its $y$ and $z$ coordinates and all co- ordinates of the second (right) $\mathrm{Ti}$ atom are relaxed to minimize the total energy. At the unstable bridge site, the energy barrier is calculated to be $Q=0.26 \mathrm{eV}$. If the left $\mathrm{Ti}$ atom can overcome this barrier, it then jumps to the adjacent hexagonal site to form a dimer with second (right) Ti atom corresponding to state II. This analysis indicates that the Ti coverage to form a $(2 \times 2)$ structure is stable and does not allow dimerization of $\mathrm{Ti}$ atoms by themselves. We also relaxed the regular $(2 \times 2)$ pattern of the adsorbed Ti atoms using a $(4$ $\times 4)$ graphene supercell, and hence lifted the possible constraints to be imposed by a relatively smaller $(2 \times 2)$ supercell. We did not obtain any tendency toward dimerization of $\mathrm{Ti}$ atoms.

An interesting reconstruction of regular $(2 \times 2)$ pattern of adsorbed $\mathrm{Ti}$ atoms has occurred when two adjacent arrays along $\vec{\eta}=-\frac{1}{2} \hat{x}+\frac{\sqrt{3}}{2} \hat{y}$ direction are displaced toward each other as shown in Fig. 5. The increased Ti-Ti coupling leads to the formation of zigzag chains on the graphene. The total energy of this chain structure is lowered by $0.65 \mathrm{eV}$ per $\mathrm{Ti}$ atom

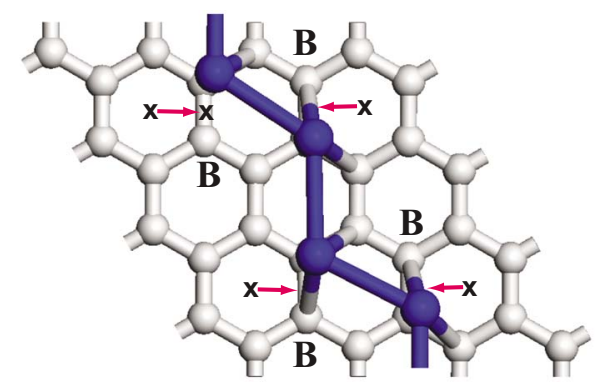

FIG. 5. (Color online) A zigzag chain of Ti atoms is formed, when two adjacent rows of $\mathrm{Ti}$ atoms are displaced from hexagonal site toward the bridge sites as shown by arrows. The system reconstructs from a $(2 \times 2)$ to a $(4 \times 4)$. 


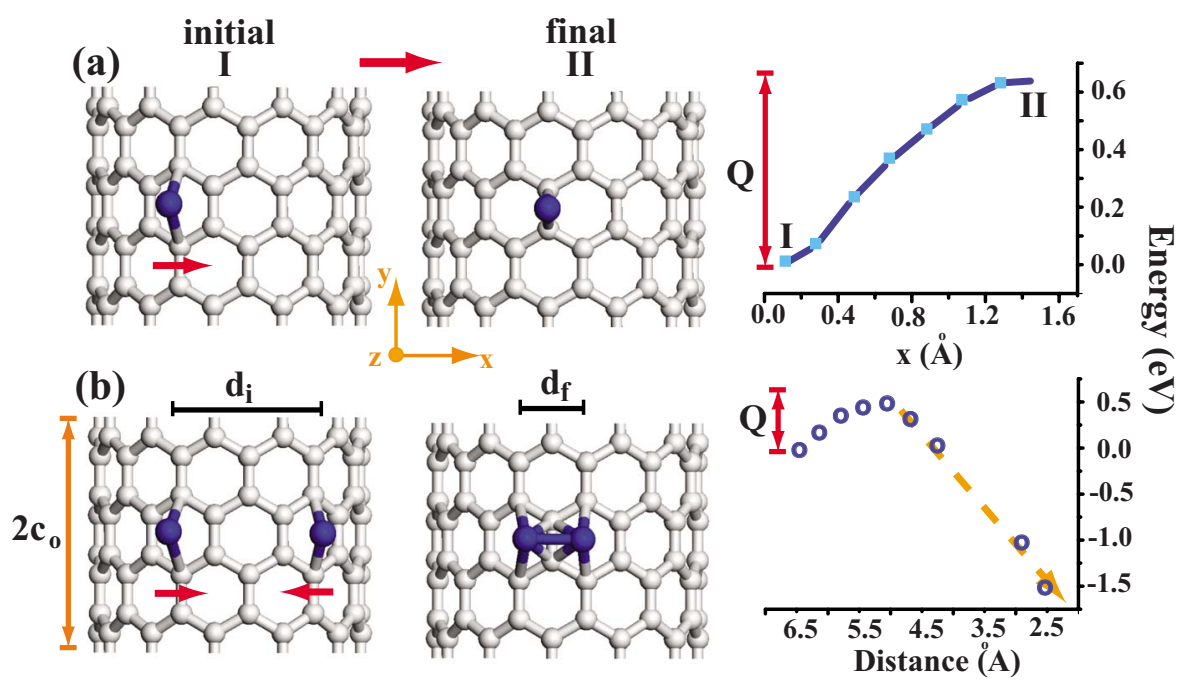

FIG. 6. (Color online) (a) Variation of total energy in the translation of a single Ti atom from the hexagonal site of the (12,0) SWNT to the bridge site and the involved energy barrier $Q$. (b) Variation of total energy and involved potential barrier from the initial state I to the final state II corresponding to $\mathrm{Ti}_{2}$ dimer adsorbed on SWNT. The barrier occurs at the center of the right bridge. Overcoming the barrier the left Ti jumps to the adjacent hexagonal site to form $\mathrm{Ti}_{2}$ with the right $\mathrm{Ti}$ atom. $d_{i}$ and $d_{f}$ are initial and final distances between two Ti atoms. $c_{0}$ is the lattice parameter of the $(12,0)$ SWNT.

relative to the uniform structure in Fig. 2(a). However, the energy barriers to form zigzag chain is $Q \simeq 0.18 \mathrm{eV}$ per Ti.

We also performed a similar analysis for the Ti atoms adsorbed on the zigzag $(12,0)$ SWNT. For the diffusion of a single $\mathrm{Ti}$ atom adsorbed at the hexagonal site of $(12,0)$ SWNT, there can be three different paths for the diffusion of Ti atom; namely, toward (i) one of the carbons, (ii) the bridge site of the $\mathrm{C}-\mathrm{C}$ bond parallel to the tube axis, and (iii) the bridge site of the tilted $\mathrm{C}-\mathrm{C}$ bond. The first (third) path has energy barrier $Q$ slightly higher (lower) than the energy barrier of the second path. As an average value we consider that the energy barrier $Q$ is calculated by minimizing the total energy relative to the $y$ and $z$ positions for each $x$ position of the Ti atom moving toward the bridge site. In these calculations, two primitive unit cells of SWNT are included in the supercell with the minimum distance between two $\mathrm{Ti}$ atoms adsorbed to adjacent supercells being $8.5 \AA$ (or $c=2 c_{0}, c_{0}$ being the optimized lattice parameter of SWNT). The energy barrier which is calculated to be $0.64 \mathrm{eV}$ did not change significantly if the underlying SWNT is relaxed. For the reason discussed above, this barrier is high enough to yield relatively low diffusion constant for an adsorbed $\mathrm{Ti}$ atom. Using potential energy in Fig. 6(a) we estimated the frequency $\nu \simeq 4 \times 10^{12} \mathrm{~s}^{-1}$. The surface diffusion constant at room temperature is $D=1.2 \times 10^{-6} \mathrm{~cm} \mathrm{~s}^{-1}$, with $D_{0}=6$ $\times 10^{4} \mathrm{~cm} \mathrm{~s}^{-1}$. We note that two Ti atoms which are placed to two adjacent hexagonal sites each corresponding to the equilibrium site of a single (isolated) $\mathrm{Ti}$ atom tend to form a dimer without energy barrier. Upon dimer formation, the total energy of the $\mathrm{Ti}_{2}+\mathrm{SWNT}$ system has lowered by $3.49 \mathrm{eV}$ relative to a free $\mathrm{Ti}$ atom and a single $\mathrm{Ti}$ is adsorbed on the SWNT.

We next address the question whether there is an energy barrier, $Q$, if two Ti atoms were adsorbed at two hexagonal sites by leaving one hexagonal site free as shown in Fig. 6(b) as initial state I. The total energy of the system described in
Fig. 6(a) as initial state is lowered by $1.69 \mathrm{eV}$ upon the adsorption of a second Ti as shown in Fig. 6(b). However, this state corresponds to a local minimum. The transition from initial state I to the final state II in Fig. 6(b) is examined by minimizing the total energy as the left $\mathrm{Ti}$ is moving toward the right bridge site. For each increment of $x$, the $y$ and $z$ coordinates of the left $\mathrm{Ti}$ and all coordinates of right Ti have been optimized. The energy barrier between initial and final states is found to be $\sim 0.45 \mathrm{eV}$ at the center of the bridge site. We found that the $\mathrm{Ti}_{2}$ dimer can form only if the left $\mathrm{Ti}$ atom overcomes this barrier and jumps to the adjacent hollow site. Upon dimerization, the total energy is lowered by $1.79 \mathrm{eV}$.

Clearly, high Ti coverage of SWNT, such as $\mathrm{C}_{2}(\mathrm{Ti})$ or $\mathrm{C}_{4}(\mathrm{Ti})$ which allow two $\mathrm{Ti}$ atoms to be placed to adjacent hollow sites with strong Ti-Ti coupling, is vulnerable to the dimerization, whereas the uniform $\mathrm{C}_{8}(\mathrm{Ti})$ coverage is stable and $\mathrm{Ti}$ atoms are prevented from dimerization by a significant energy barrier of $0.45 \mathrm{eV}$. This result clearly demonstrates that the argument by Sun et al. ${ }^{22}$ based on the minimum energy configuration is not by itself sufficient to decide on the final stable configuration. Transition from the high energy to the low energy configuration may be hindered by an energy barrier. ${ }^{45}$

\section{CONCLUSION}

In this study we examined the hydrogen storage capacity of $\mathrm{Sc}, \mathrm{Ti}$, and $\mathrm{V}$ adsorbed to various carbon-based structures in different geometries and dimensionalities; namely, linear C chain, graphene, and $(12,0)$ zigzag SWNT. These structures can bind $\mathrm{Sc}, \mathrm{Ti}$, and $\mathrm{V}$ with comparable and significant chemisorption energy. Each adsorbed Ti can bind up to four or five $\mathrm{H}_{2}$ molecules depending on the character of the bond it forms with underlying carbon-based structure. In the case 
of Ti- $\mathrm{C}_{n}$-Ti linear chain, $\mathrm{H}_{2}$ uptake capacity is higher than that of Ti-SWNT due to the bond between Ti and adjacent single $\mathrm{C}$ atom. Sc and $\mathrm{V}$ atoms display also similar behavior as far as the number of $\mathrm{H}_{2}$ they can hold, except the situation where Sc or V adsorbed on the external side of SWNT. While each Ti on the $(12,0)$ SWNT can bind up to four $\mathrm{H}_{2}$, Sc and $\mathrm{V}$ can hold even five $\mathrm{H}_{2}$ molecules for a specific absorption geometry. The storage capacity of these carbonbased structures, which are decorated by light TM atoms, is higher than generally accepted requirement of $6 \mathrm{wt} \%$ set for practical applications. The calculated average binding energies of $\mathrm{H}_{2}$ usually range between 0.3 and $0.5 \mathrm{eV}$, which are convenient to store hydrogen molecules at room temperature and to discharge them upon heating. Estimated desorption temperatures indicate that carbon-based structures are convenient as far as recycling is concerned. This is a desirable characteristic for long term recycling of the storage medium. Since the bonding is molecular and therefore weak, we expect to see very fast kinetics in the course of the absorption and/or desorption process. We also showed that TM atoms adsorbed inside SWNT can bind multiple hydrogen molecules, which, in turn, may increase the storage capacity, if the internally adsorbed TM atoms are not vulnerable to clustering in the course of recycling. Finally, we found that the $\mathrm{Ti}_{2}$ dimer can form only if the distance between two adsorbed Ti atoms is small and hence allows strong coupling between them at high coverage. However, in spite of the fact that the dimer has lower total energy, its formation is hindered by a significant energy barrier between distant $\mathrm{Ti}$ atoms. This result shows that the transition state analysis is needed to decide whether a low energy configuration can occur.

\section{ACKNOWLEDGMENTS}

This work was supported by TÜBİTAK under Grant No. TBAG-104T536. T.Y. acknowledges partial support from DOE under BES Grant No. DE-FG02-98ER45701.

*ciraci@fen.bilkent.edu.tr

${ }^{1}$ R. Coontz and B. Hanson, Science 305, 957 (2004).

${ }^{2}$ This is defined as the ratio of the total mass of absorbed hydrogens to the mass of absorbing structure including also the mass of hydrogens.

${ }^{3}$ G. W. Crabtree, M. S. Dresselhaus, and M. V. Buchanan, Phys. Today 57 (12), 39 (2004).

${ }^{4}$ A. C. Dillon, K. M. Jones, T. A. Bekkendahl, C. H. Kiang, D. S. Bethune, and M. J. Heben, Nature (London) 386, 377 (1997).

${ }^{5}$ S. P. Chan, G. Chen, X. G. Gong, and Z. F. Liu, Phys. Rev. Lett. 87, 205502 (2001).

${ }^{6}$ K. Tada, S. Furuya, and K. Watanabe, Phys. Rev. B 63, 155405 (2001).

${ }^{7}$ P. Dubot and P. Cenedese, Phys. Rev. B 63, 241402(R) (2001).

${ }^{8}$ O. Gulseren, T. Yildirim, and S. Ciraci, Phys. Rev. Lett. 87, 116802 (2001).

${ }^{9}$ E.-C. Lee, Y.-S. Kim, Y.-G. Jin, and K. J. Chang, Phys. Rev. B 66, 073415 (2002).

${ }^{10}$ Y. Miura, H. Kasai, W. A. Dino, H. Nakanishi, and T. Sugimoto, J. Appl. Phys. 93, 3395 (2003).

${ }^{11}$ G. Lu, H. Scudder, and N. Kioussis, Phys. Rev. B 68, 205416 (2003).

${ }^{12}$ W.-Q. Deng, X. Xu, and W. A. Goddard, Phys. Rev. Lett. 92, 166103 (2004).

${ }^{13}$ S. Dag, Y. Ozturk, S. Ciraci, and T. Yildirim, Phys. Rev. B 72, 155404 (2005).

${ }^{14}$ T. Yildirim and S. Ciraci, Phys. Rev. Lett. 94, 175501 (2005).

${ }^{15}$ Y. Zhao, Yong-Hyun Kim, A. C. Dillon, M. J. Heben, and S. B. Zhang, Phys. Rev. Lett. 94, 155504 (2005).

${ }^{16}$ T. Yildirim, J. Iniguez, and S. Ciraci, Phys. Rev. B 72, 153403 (2005).

${ }^{17}$ B. Bogdanovic, M. Felderhoff, S. Kaskel, A. Pommerin, K. Schlichte, and F. Schuth, Adv. Mater. (Weinheim, Ger.) 15, 1012 (2003).

${ }^{18}$ T. Yildirim and M. R. Hartman, Phys. Rev. Lett. 95, 215504 (2005).

${ }^{19}$ Y. Zhao, A. C. Dillon, Y.-H. Kim, M. J. Heben, and S. B. Zhang, Chem. Phys. Lett. 425, 273 (2006).

${ }^{20}$ N. Akman, E. Durgun, T. Yildirim, and S. Ciraci, J. Phys.: Condens. Matter 18, 9509 (2006).

${ }^{21}$ H. Lee, W. I. Choi, and J. Ihm, Phys. Rev. Lett. 97, 056104 (2006).

${ }^{22}$ Q. Sun, Q. Wang, P. Jena, and Y. Kawazoe, J. Am. Chem. Soc. 127, 14582 (2005).

${ }^{23}$ E. Durgun, S. Ciraci, W. Zhou, and T. Yildirim, Phys. Rev. Lett. 97, 226102 (2006).

${ }^{24}$ M. C. Payne, M. P. Teter, D. C. Allen, T. A. Arias, and J. D. Joannopoulos, Rev. Mod. Phys. 64, 1045 (1992).

${ }^{25}$ Numerical computations have been carried out by using VASP software: G. Kresse and J. Hafner, Phys. Rev. B 47, R558 (1993); G. Kresse and J. Furthmuller, ibid. 54, 11169 (1996).

${ }^{26}$ P. Hohenberg and W. Kohn, Phys. Rev. 136, B864 (1964); W. Kohn and L. J. Sham, ibid. 140, A1133 (1965).

${ }^{27}$ D. Vanderbilt, Phys. Rev. B 41, R7892 (1990).

${ }^{28}$ J. P. Perdew, J. A. Chevary, S. H. Vosko, K. A. Jackson, M. R. Pederson, D. J. Singh, and C. Fiolhais, Phys. Rev. B 46, 6671 (1992).

${ }^{29}$ M. Methfessel and A. T. Paxton, Phys. Rev. B 40, 3616 (1989).

${ }^{30}$ H. J. Monkhorst and J. D. Pack, Phys. Rev. B 13, 5188 (1976).

${ }^{31}$ G. Roth and H. Fischer, Organometallics 15, 5766 (1996).

${ }^{32}$ X. Zhao, Y. Ando, Y. Liu, M. Jinno, and T. Suzuki, Phys. Rev. Lett. 90, 187401 (2003).

${ }^{33}$ S. Tongay, R. T. Senger, S. Dag, and S. Ciraci, Phys. Rev. Lett. 93, 136404 (2004); S. Tongay, S. Dag, E. Durgun, R. T. Senger, and S. Ciraci, J. Phys.: Condens. Matter 17, 3823 (2005); R. T. Senger, S. Tongay, S. Dag, E. Durgun, and S. Ciraci, Phys. Rev. B 71, 235406 (2005), and references therein.

${ }^{34}$ The binding energy is obtained by using the expression $E_{b}$ $=E_{T}[\mathrm{Ti}]+E_{T}\left[\mathrm{C}_{5}\right]-E_{T}\left[\mathrm{Ti}-\mathrm{C}_{5}\right]$ in terms of the total energies of free $\mathrm{Ti}$ atom, free $\mathrm{C}_{5}$, and the total energy of $\mathrm{Ti}-\mathrm{C}_{5}$, which are calculated by optimizing atomic structure through spin-polarized GGA in a supercell. $E_{b}>0$ indicates a stable structure. 
${ }^{35}$ A. K. Geim and K. S. Novoselow, Nature (London) 6, 183 (2007).

${ }^{36}$ Note that significant Ti-Ti coupling energy due to decreased Ti-Ti distance is subtracted to find the binding energy between a single $\mathrm{Ti}$ and graphene.

${ }^{37}$ E. Durgun, S. Dag, V. M. K. Bagci, O. Gülseren, T. Yildirim, and S. Ciraci, Phys. Rev. B 67, 201401(R) (2003).

${ }^{38}$ E. Durgun, S. Dag, S. Ciraci, and O. Gülseren, J. Phys. Chem. B 108, 575 (2004).

${ }^{39}$ Modern Coordination Chemistry: The Legacy of Joseph Chatt, edited by G. J. Leigh and N. Winterton (Royal Society of Chemistry, Cambridge, 2002).

${ }^{40}$ Metal Dihydrogen and Bond Complexes Structure, Theory, and Reactivity, edited by G. J. Kubas (Kluwer, Dordrecht, 2001).

${ }^{41} \mathrm{We}$ were able to determine appropriate geometry which permits the adsorption of five $\mathrm{H}_{2}$ on Sc and V on SWNT. Nevertheless, the binding energy of five $\mathrm{H}_{2}$ is weaker than that of four $\mathrm{H}_{2}$. However, we were unable to find such a configuration that allows the absorption of five $\mathrm{H}_{2}$ for Ti on SWNT.

${ }^{42}$ Handbook of Chemistry and Physics, 75th ed., edited by D. R. Lide (CRC, New York, 1994).

${ }^{43}$ We note that $T_{D_{L}}, T_{D}$, and $T_{D_{H}}$ given in Table II have to be taken as rough estimates for the desorption temperature where the desorption takes place due to the definition of $\Delta S$.

${ }^{44}$ S. Dag, E. Durgun, and S. Ciraci, Phys. Rev. B 69, 121407(R) (2004).

${ }^{45}$ We note that we performed the same calculations using a single cell with $c=c_{0}$. Because of significant Ti-Ti coupling among Ti atoms between adjacent supercells, we obtained that $Q$ in diffusion decreases to $0.58 \mathrm{eV}$, and the binding energy of a single atom increases to $1.76 \mathrm{eV}$. 